\title{
Rol de la multimodalidad de imagen en insuficiencia cardíaca
}

\author{
Dres. Lucía Florio, Gabriel Parma
}

\begin{abstract}
Resumen
En la presente revisión los autores abordan el concepto de multimodalidad de imagen a través del conocimiento de las diferentes técnicas de imagen cardiológica y el aporte de cada una de ellas al diagnóstico de la insuficiencia cardíaca para así construir un esquema de secuencias de estudios o complementariedad de los mismos por separado o en conjunto (técnicas de fusión) aplicable a los pacientes con insuficiencia cardíaca confirmada o sospechada.

Se analizan someramente los fundamentos técnicos y clínicos de cada técnica, los aportes de la multimodalidad al diagnóstico de insuficiencia cardíaca encarando las perspectivas futuras de esta metodología.

Al final del capítulo se ofrece un resumen conceptual para la aplicación práctica de los conceptos analizados en el mismo.

Palabras clave: $\quad$ IMAGEN MULTIMODAL

DIAGNÓSTICO

INSUFICIENCIA CARDÍACA
\end{abstract}

\section{Role of imaging multimodality in heart failure}

\section{Summary}

In this review the authors intend to approach to the concept of multimodality of images, through the knowledge of the different techniques and the contribution of each of them to the diagnosis of heart failure, in order to construct a scheme of studies sequences or complementarity of them, either separately or together (fusion techniques) applicable to the patient with confirmed or suspected heart failure.

They briefly analyze the technical and clinical bases of each technique, the contributions of multimodality to the diagnosis of heart failure facing the future perspectives of this methodology.

At the end of the chapter a conceptual summary is offered for the practical application of the concepts analyzed above.

Key words:

\section{MULTIMODALITY IMAGING \\ DIAGNOSIS \\ HEART FAILURE}

\section{Introducción}

La evaluación por técnicas de imagen tiene un rol muy importante en la valoración del paciente con insuficiencia cardíaca (IC). Si bien la ecocardiografía transtorácica (ETT) bidimensional sigue siendo el estudio central en la evaluación estructural y funcional del corazón, se han ido incorporando diferentes técnicas que complementan y amplían la información en este grupo de pacientes ${ }^{(1)}$.

En cardiología, las diferentes técnicas imagenológicas pueden ser utilizadas en forma independiente, integradas (técnicas de fusión), o en forma sucesiva y coordinada, ya sea para definir la etiología de una cardiopatía, la extensión de isquemia o de daño miocárdico, o bien para indicación de medidas tera- péuticas o evaluación de respuesta a las mismas. A este enfoque se le ha denominado multimodalidad de imagen (MI).

En pacientes con clínica de IC, la MI ofrece la oportunidad de obtener una adecuada información sobre la función cardíaca global o sectorial, la viabilidad miocárdica, la perfusión miocárdica o la anatomía coronaria.

En este capítulo se revisará el papel de la MI en los pacientes con IC, para ello se abordarán tres aspectos de la misma:

- Fundamentos de cada técnica en la evaluación de la IC.

- Aportes de la MI al diagnóstico de IC.

- Perspectivas futuras. 


\section{I) Fundamentos de cada técnica}

\section{Ecocardiografía}

La ETT evalúa la estructura y los flujos cardíacos a través de ondas de ultrasonido, ya sea por medio del ecocardiograma bidimensional o tridimensional, del Doppler de onda continua o pulsada, del Doppler color, tisular o por strain y strain rate.

Es la técnica de imagen de primera elección en la evaluación de la IC dada su inocuidad, amplia disponibilidad y bajo costo(1). Permite evaluar la función sistólica y diastólica de ambos ventrículos, así como analizar los aparatos valvulares, el pericardio y otras eventuales causas de IC. Dado que la evaluación de las patologías valvulares excede el alcance de este capítulo solo se analizará la utilidad de la ETT en la valoración de la función de ambos ventrículos.

\subsection{Función sistólica ventricular izquierda}

La valoración de la función sistólica del ventrículo izquierdo (VI) es expresada, principalmente, través de la fracción de eyección (FEVI), que representa el cociente entre el volumen de eyección y el volumen diastólico final del VI. La FEVI puede ser estimada en forma subjetiva por un operador experimentado o a través de cálculos de volumen partiendo del área de un corte transversal (método de área-longitud) o mediante la suma de discos superpuestos (método de Simpson modificado). Ambos métodos presentan limitaciones dado el requerimiento de adecuadas ventanas apicales, buena definición del borde endocárdico y asumir una figura geométrica en su cálculo $^{(1-4)}$.

La Sociedad Americana de Ecocardiografía (ASE) y la Asociación Europea de Imagen Cardiovascular (EAIC) consensuaron considerar FEVI normal un valor $\geq 53 \%$, levemente disminuida entre $41 \%$ y $52 \%$, moderadamente disminuida entre $30 \%$ y $40 \%$ y severa $<30 \%{ }^{(2)}$. Como se verá más adelante, esta estadificación es orientativa pero no coincide con la clasificación que se hace de la IC según la FEVI.

La función contráctil ventricular es un proceso complejo, dependiente de fenómenos electromecánicos y variaciones en la pre y poscarga del VI, por lo cual la FEVI puede resultar insuficiente para la valoración integral de la misma.

La función ventricular puede ser evaluada también por parámetros hemodinámicos, como el volumen de eyección y el gasto cardíaco (GC). Estos son fácilmente calculables con la medición del diámetro del tracto de salida del VI (TSVI) y la obtención del Doppler pulsado a nivel del mismo. Se puede ampliar la valoración hemodinámica no invasiva con estimación de las presiones de arteria pulmonar, las resistencias vasculares sistémica y pulmonar, y las presiones de llenado del VI y del ventrículo derecho (VD) con aceptable reproducibilidad ${ }^{(1,2)}$.

La ecocardiografía tridimensional, con una reconstrucción automática en tres dimensiones a partir de una única adquisición apical, resulta un método rápido, reproducible y menos operador-dependiente; sin embargo, es de limitada disponibilidad en los laboratorios de ecocardiografía $\mathrm{y}$, por otra parte, si se compara con la cardiorresonancia (CR) (gold standard para el cálculo volumétrico) se observa una subestimación de los volúmenes del $\mathrm{VI}^{(2,5-9)}$.

El Doppler tisular y el estudio de la deformación miocárdica mediante el método de strain son otros parámetros útiles para la valoración de la función sistólica del VI más allá de la FEVI y, muy especialmente, para la detección precoz de disfunción sistólica ${ }^{(1,2)}$. La velocidad sistólica del Doppler tisular (onda s') a nivel del anillo mitral lateral y septal basal tienen una buena correlación con la FEVI. Por otra parte, en pacientes con IC y FEVI preservada puede detectarse una reducción en la onda s' (menor a $4,5 \mathrm{~cm} / \mathrm{s}$ ) como elemento de disfunción sistólica precoz ${ }^{(1,2,10,11)}$.

La ETT de rastreo de señales puede analizar la mecánica del músculo mediante índices de deformación y torsión. Se basa en el rastreo espacial de una unidad funcional formada por varias señales acústicas generadas en la ETT bidimensional en escala de grises que se denominan espículas. Dichas espículas se mueven durante el ciclo cardíaco (acercándose en sístole y alejándose en diástole), permitiendo calcular la deformación miocárdica sistólica ${ }^{(2)}$.

La deformación se puede analizar en los ejes longitudinal, radial y circunferencial, aunque solo el longitudinal ha demostrado aplicabilidad clínica. Se obtiene una valoración cuantitativa de la función sistólica, independiente del ángulo y movimiento del corazón (strain), y de la velocidad de esta deformación (strain rate) $)^{(12,13)}$.

La deformación longitudinal mide la distorsión miocárdica de las fibras dirigidas desde la base hacia el ápex donde predominan ampliamente las fibras subendocárdicas (las primeras en afectarse habitualmente) pudiendo identificar disfunción sistólica incluso en estado subclínico ${ }^{(2,12-17)}$. El promedio de la deformación longitudinal en las tres proyecciones apicales (dos, tres y cuatro cámaras) es la deformación longitudinal global o strain longitudinal global (SLG); considerado el parámetro de mayor rendimiento en la búsqueda de disfunción sistólica precoz. 
La caída del SLG del VI (SGLVI) es un parámetro pronóstico independiente, y se propone como índice de evaluación sistemática en algunos pacientes, por ejemplo en aquellos sometidos a quimioterapia o con estenosis aórtica severa asintomática $^{(2,17)}$. La presencia de deformación longitudinal muy baja (menor de -7\%) y reducción de la FEVI se asocian a un riesgo mayor de eventos cardiovasculares al año ${ }^{(18,19)}$.

La técnica de SLG tiene algunas limitaciones: escasa disponibilidad, dependencia de una adecuada ventana ultrasónica, variabilidad según equipos y software disponibles y una demostrada variabilidad interobservador $(2,13,17)$.

Las guías de estandarización de medidas ecocardiográficas de la Sociedad Europea de Cardiología (ESC) recomiendan el SLGVI bidimensional como una herramienta reproducible y factible para uso clínico y proponen el valor pico en el rango de -20\% como parámetro normal, siendo anormal un hallazgo de valores absolutos inferiores ${ }^{(2)}$.

\subsection{Función diastólica ventricular izquierda}

La evaluación de la función diastólica es parte integral de la evaluación ecocardiográfica de los pacientes con IC.

La ETT permite estimar la presión diastólica final del VI (PDFVI) a través de varios parámetros: patrón de llenado mitral con Doppler pulsado (relación $\mathrm{E} / \mathrm{A}$ ); volumen de aurícula izquierda; relación de velocidades del anillo mitral (onda e' de Doppler tisular) con onda E del flujo transmitral; velocidad de insuficiencia tricuspídea; patrón de flujo en las venas pulmonares mediante Doppler pulsado. También se puede estimar a partir de la diferencia entre la presión arterial sistólica sistémica y el gradiente máximo de la regurgitación mitral (de estar presente) $)^{(1,20,21)}$.

El aumento de volumen de la AI puede reflejar un aumento de la PDFVI, aceptándose un volumen mayor a $34 \mathrm{ml} / \mathrm{m}^{2}$ como posible expresión de disfunción diastólica. En la fase inicial de la disfunción diastólica el volumen de la AI puede ser normal pero no la funcionalidad de la misma, por lo que podría ser útil el análisis de la deformación de la AI por método de strain. Esto último tiene, actualmente, escasa aplicabilidad clínica dada su restringida disponibilidad y la escasa evidencia que permita incluirla en los algoritmos diagnósticos, pero se propone como método futuro de evaluación de estos pacientes $^{(20-24)}$.

Los parámetros de mayor sensibilidad y especificidad en la estimación de la PDFVI son la velocidad protodiastólica del flujo mitral (onda E) y la del anillo mitral (e') obtenida por Doppler tisular. Elín- dice E/e' es el más utilizado pues ha demostrado una relación directa con la PDFVI y presión de la AI medidas en forma invasiva tanto en pacientes con FEVI preservada como descendida ${ }^{(21,25-27)}$.

\subsection{Evaluación de ventrículo derecho}

El VD tiene una importante connotación pronóstica en el contexto de la falla ventricular izquierda, por lo cual debe evaluarse siempre que sea posible.

La evaluación morfológica del VD a través de la ETT resulta dificultosa debido a la imposibilidad de asumir una figura geométrica simple para su análisis, con planos poco estandarizados y reproducibles, y la presencia de hipertrabeculación endocárdica. Sin embargo, es un método accesible y útil si es realizado por un técnico experimentado que respete las recomendaciones para sus medidas ${ }^{(1,2)}$.

Las mediciones del VD se han estandarizado en enfoque apical de cuatro cámaras (en una proyección centrada y no oblicua) a nivel basal y medio, considerando normales los diámetros que no excedan los 42 y $36 \mathrm{~mm}$, respectivamente. También se estandarizaron las medidas en el tracto de salida del VD, marcando un punto de referencia proximal de $36 \mathrm{~mm}$ y distal de $28 \mathrm{~mm}$ medidos en eje corto de la base. Se sigue recomendando la valoración cualitativa del VD, pautando que en apical cuatro cámaras y en forma centrada las dimensiones del VD no deben superar los dos tercios de las del $\mathrm{VI}^{(2)}$.

En cuanto a la valoración funcional, las medidas volumétricas tienen elevada variabilidad y no se recomiendan como método de evaluación sistemática. La distribución de las fibras (orientación longitudinal, desde la punta hacia la base, con un descenso longitudinal hacia la base en sístole) hacen que las mediciones no volumétricas sean las más adecuadas para la evaluación de la función sistólica.

Las mediciones no volumétricas son cuatro:

- Excursión sistólica del plano del anillo tricuspídeo (TAPSE, por su sigla en inglés). Midiendo en modo $\mathrm{M}$ la distancia que recorre el anillo tricuspídeo lateral en sístole tomándose como punto de corte mínimo $17 \mathrm{~mm}$.

- Medición de la velocidad del desplazamiento anular tricuspídeo a través de la onda s' del Doppler tisular. Es el método de mayor sensibilidad. El punto de corte es de $9,5 \mathrm{~cm} / \mathrm{s}$.

- Índice de rendimiento miocárdico (índice de TEI): es una medida de intervalos temporales entre el flujo tricuspídeo y pulmonar, siendo normal un valor menor a 0,5 .

- Medida del SLG del VD, con las mismas características que las pautadas para el VI (en apical cuatro cámaras) tomando exclusivamente la pared libre. Presenta las mismas fortalezas y debi- 
lidades señaladas para el VI. Punto de corte aceptado en $-20 \%{ }^{(2)}$.

\section{Cardiorresonancia magnética}

La cardiorresonancia (CR) es una técnica que se basa en la interpretación de imágenes que surgen del comportamiento de los átomos corporales sometidos a las fuerzas de un campo magnético; no utiliza radiaciones ionizantes y es muy importante en la valoración actual de la IC, permitiendo una evaluación integral anátomo-funcional de ambos ventrículos con una "caracterización tisular" del tejido miocárdico y, en forma accesoria, la evaluación de todo el complejo cardiovascular mediante el análisis de pericardio, aparatos valvulares y grandes vasos, tanto en su morfología como en su función.

Para comprender la utilidad e indicación de la CR es imprescindible conocer las secuencias de adquisición y su utilidad. Se pueden agrupar en: secuencias de valoración de la función sistólica; de análisis de la "caracterización tisular" y de análisis de grandes vasos ${ }^{(1,28-30)}$. A los efectos de cumplir los objetivos de este capítulo se describirán solamente los dos primeros grupos.

\subsection{Análisis de secuencias}

2.1.1. Secuencias de valoración de la función sistólica La CR es el estándar de referencia para la evaluación de la función cardíaca.

Dentro de las secuencias de análisis existen tres: secuencia cine, que permite la medición exacta de volúmenes y fracción de eyección del VI y VD; de contraste de fase, que permite la medición del volumen sistólico, y de tagging, que permite el análisis de la mecánica ventricular.

- 2.1.1.1. Secuencia cine

La secuencia de este tipo más utilizada es la denominada, por su sigla en inglés, SSFP (Steady-state free precession). Es una secuencia de "sangre blanca" obtenida en movimiento (modo cine). Tiene una excelente resolución de contraste entre la sangre (hiperintenso) y el miocardio con una alta resolución temporal (adquiriendo una imagen cada 20-40 $\mathrm{ms}$ ), todo lo cual la convierte en la técnica de elección para el análisis morfológico y funcional del VI y VD.

Se pueden obtener cortes en tiempo real y en forma sistematizada tanto en eje corto, como apical, obteniéndose, de forma automática y exacta, volúmenes y fracción de eyección de ambas cavidades independientemente de cualquier forma geométrica de cálculo ${ }^{(31)}$.

Se han publicado tablas con valores de referencia de volúmenes, función y masa del VI y VD para una población sana. Sin embargo, a diferencia de la ecocardiografía, aún no existe consenso ni recomendaciones sobre la graduación de la disfunción por este método $^{(29)}$.

- 2.1.1.2. Secuencia de contraste de fase

La secuencia de contraste de fase consta de una adquisición en forma simultánea de una serie de cine y de velocidad, reflejando el flujo sanguíneo mediante escala de grises ${ }^{(30,31)}$.

Con esta secuencia se puede calcular en forma directa el volumen sistólico del VI y VD, el GC y el valor del $\mathrm{QP} / \mathrm{QS}$.

Se puede obtener información cuantitativa, en tiempo real, del flujo sistólico a nivel de la aorta y de la arteria pulmonar y sus ramas y se puede, también, cuantificar, en forma directa o indirecta, las regurgitaciones de las válvulas sigmoideas y auriculoventriculares.

\section{- 2.1.1.3 Secuencia de tagging}

Es una secuencia que consiste en la anulación de la señal en el momento de la captación de la imagen a través de pulsos de presaturación. Como consecuencia se obtiene una proyección de líneas negras en rejilla sobre el miocardio. Estas rejillas definen la posición del miocardio en movimiento permitiendo una mejor evaluación de las alteraciones de la motilidad sectorial y de la deformación miocárdica( ${ }^{(30)}$.

\subsubsection{Secuencias de caracterización tisular}

Las secuencias de caracterización tisular derivan de la relajación de los protones. Mediante la elección de cada una y el conocimiento de lo que sucede en ellas, se puede evaluar qué está sucediendo con el tejido miocárdico.

Para su sistematización se clasificarán en:

a) Secuencias de sangre negra.

b) Secuencias con contraste (gadolinio).

c) Secuencias de mapeo miocárdico.

- a) Secuencias de sangre negra

Los protones de hidrógeno giran sobre sí mismos en un movimiento conocido como "espín”, que se altera cuando se someten al campo magnético de la CR; según el tiempo que tarden en relajarse al ser liberados del campo externo, se obtienen las secuencias de espín eco; la sangre resulta hipointensa (negra), produciéndose un excelente contraste entre esta y el miocardio.

Modificando parámetros de la secuencia se pueden obtener imágenes potenciadas en $\mathrm{T} 1$ o T2 $2^{(31)}$. La secuencia potenciada en T1 es útil para el estudio morfológico del corazón y grandes vasos, y para la caracterización tisular, al destacar los tejidos o sustancias que contrastan con el miocardio (grasa, exudados y hemorragias). La secuencia potenciada en 
T2 es la de elección para evidenciar contenido de agua, pues esta se mostrará hiperintensa; es, por tanto, útil para detectar edema miocárdico (miocarditis).

- b) Secuencias con contraste (gadolinio)

El quelato de gadolinio es un contraste paramagnético que acorta el tiempo de relajación de T1 del tejido captante. Las secuencias que derivan de este fenómeno son las de perfusión, realce precoz y realce tardío.

Las secuencias de perfusión se obtienen antes, durante y después de la administración del bolo inicial de gadolinio y permiten analizar la perfusión miocárdica en reposo. Se puede realizar con estrés farmacológico (adenosina) y generar un estudio funcional de isquemia. Las zonas de hipoperfusión se verán como áreas de señal hipointensa a diferencia del miocardio con perfusión normal que se visualiza hiperintenso.

Las secuencias de realce precoz consisten en la misma secuencia de espín eco al minuto posgadolinio. En esta se van a remarcar las áreas de miocardio "hiperémico" por aumento de la permeabilidad tisular (secuencia importante en la valoración de la miocarditis).

Las secuencias de realce tardío se basan en el hecho de que el gadolinio es un contraste de depósito extracelular, por lo que el miocardio sano (con poco espacio extracelular entre miocitos) lo "lava" rápidamente, mientras que el dañado/necrosado (fibrosis) lo hace mucho más lento. Para obtener el contraste exacto entre el miocardio sano y dañado se debe esperar entre 7 y 15 minutos $^{(31)}$.

Como veremos más adelante la simple presencia de fibrosis en el contexto de IC es un factor pronóstico muy importante.

Muchas enfermedades que afectan al miocardio tienen un tipo de distribución específica del gadolinio, lo que hace que la secuencia de realce tardío sea importante en la valoración etiológica de un paciente con $\mathrm{IC}^{(29)}$.

La tipificación clásica de dicha distribución distingue la isquémica de la no isquémica. En la isquémica (generada por necrosis por afectación vascular coronaria) se ve una distribución subendocárdica (de distribución vascular); en la de tipo no isquémica una distribución no subendocárdica (subepicárdica o intramiocárdica, o ambas).

- c) Secuencias de mapeo miocárdico

Las secuencias de mapeo miocárdico se encuentran aún en fase de desarrollo. Se obtienen mediante la búsqueda de mapas paramétricos de la relajación que permiten cuantificar el grado de lesión tisular buscando factores infiltrantes o fibrosis ${ }^{(32)}$.
La fortaleza que tiene la secuencia de mapeo en relación con la de realce tardío es poder demostrar estos patrones en estadios muchos más precoces y con mayor sensibilidad. También mediante esta secuencia se puede realizar el cálculo de fracción de volumen extracelular pudiendo detectar colágeno en ausencia de edema o infiltración.

Por tanto, este tipo de secuencia se propone como técnica prometedora en la evaluación diagnóstica y pronóstica tanto en IC con FEVI preservada como disminuida, con las implicancias terapéuticas que esto asume ${ }^{(32)}$.

Existe otra secuencia de mapas paramétricos que es la llamada T2* (T2 estrella) que ha marcado un punto de inflexión en el diagnóstico de las miocardiopatías por depósito de hierro ${ }^{(1,32)}$.

\subsection{Limitaciones y contraindicaciones de la cardiorresonancia}

Las limitaciones de la CR están dadas por su escasa disponibilidad con necesidad de una logística compleja en cuanto a recursos materiales y entrenamiento del personal técnico. También los requisitos para un estudio de adecuada calidad limitan la población que pueda acceder al mismo, ya que se requiere que los pacientes puedan soportar el decúbito supino durante al menos 30 minutos; que presenten ritmo sinusal sin extrasistolía frecuente, y que puedan realizar apneas de al menos 10 segun$\operatorname{dos}^{(1,28,29)}$

Las contraindicaciones de la CR son las siguientes:

- Presencia de marcapasos, desfibriladores o cualquier otro dispositivo no compatible para CR por ferromagnetismo.

- Presencia de clips de aneurisma intracraneal o elementos metálicos intraoculares o coclear.

- Hábito corporal que exceda las restricciones propias del equipo de resonancia magnética (peso mayor a $150 \mathrm{~kg}$, circunferencia corporal mayor al diámetro del tubo del resonador).

- Claustrofobia.

- Tasa de filtración glomerular (TFG) < $30 \mathrm{ml} / \mathrm{min}$ (si se va a administrar gadolinio).

\section{Tomografía cardíaca}

La tomografía cardíaca (TC) es una técnica de imagen cardíaca que toma el fundamento de cualquier estudio tomográfico y que tiene como ventajas la rápida adquisición y su alta resolución espacial para la reconstrucción tridimensional, con la desventaja que utiliza radiaciones ionizantes y contraste yodado.

La TC tiene requisitos que marcan una limitación en la accesibilidad a la misma: requiere de sin- 


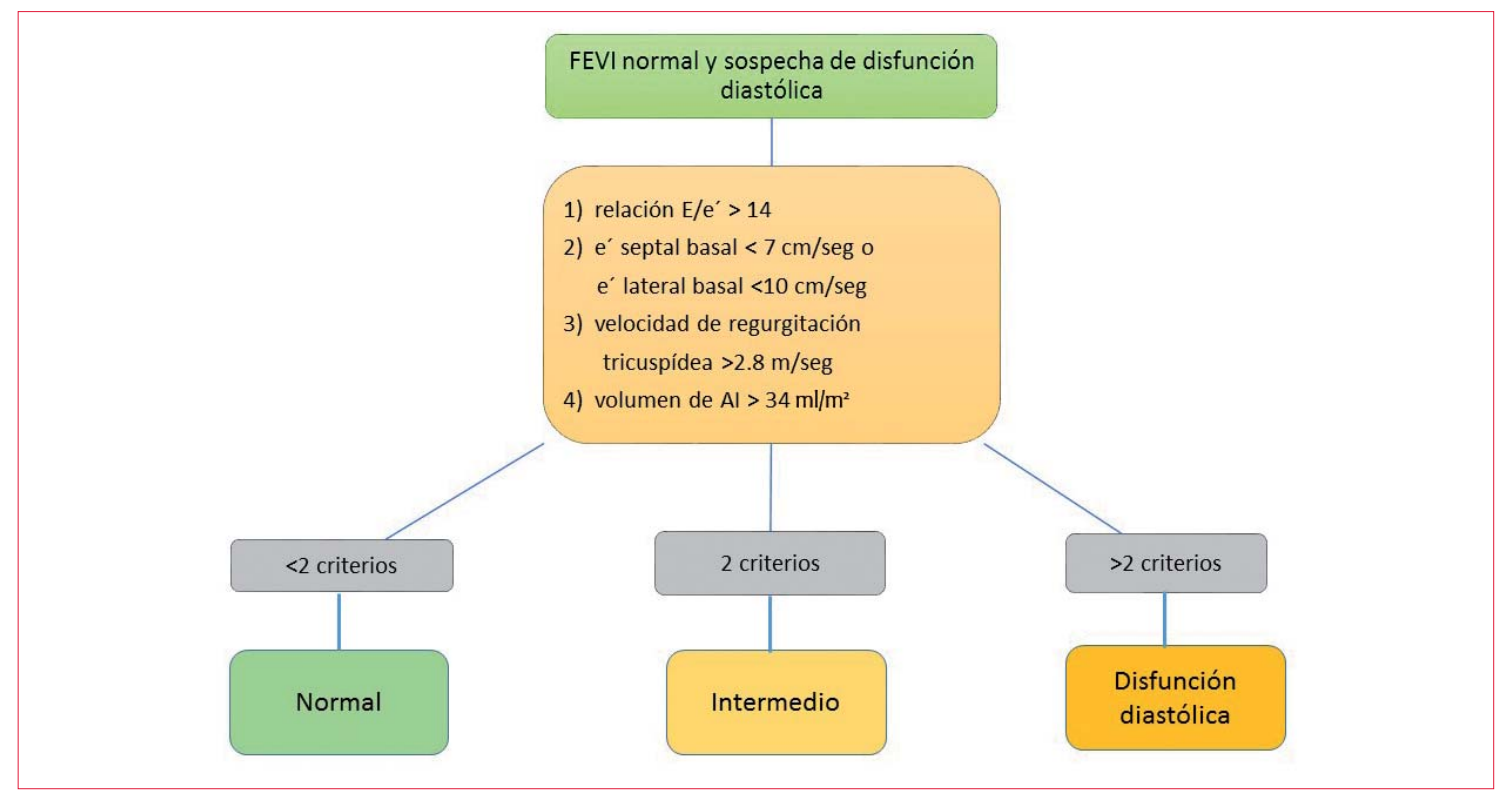

Figura 1. Criterios ecocardiográficos para definir función diastólica en pacientes con insuficiencia cardíaca con FEVI preservada.

cronización con el electrocardiograma (ECG), tomando RR regulares con una frecuencia cardíaca menor a 60/min, y necesita de un tomógrafo con multidetectores para su adquisición (al menos 64).

Es una técnica importante en MI de la IC para valorar la presencia de enfermedad coronaria y permite el estudio de la morfología y función en aquellos pacientes en los cuales no se pueda obtener esta información por otros métodos recomendados. Tiene, además, un rol en la evaluación morfológica previa a tratamientos específicos de la IC, como la valoración de venas coronarias en pacientes candidatos a terapia de resincronización, valoración estructural en pacientes candidatos a implante de válvula percutánea y análisis morfológico en cardiopatías congénitas complejas.

La fortaleza de la coronariografía por TC radica en la capacidad para descartar enfermedad coronaria en forma no invasiva, con un valor predictivo negativo cercano a $100 \%(33)$.

En cuanto a la valoración del miocardio existen protocolos que permiten obtener imágenes en forma continua a lo largo de un ciclo y luego, automáticamente, generar una imagen en movimiento muy similar a las adquiridas por ecocardiografía y resonancia, permitiendo la valoración anatómica y funcional del VI o VD. De todas maneras, su lugar es como técnica alternativa a estas, dado el uso de radiación ${ }^{(1,34)}$.
II) Aportes de la multimodalidad de imagen en la insuficiencia cardíaca

Las técnicas de imagen, en la evaluación de la IC, deben realizarse con el concepto de MI. Esto significa que todas las técnicas pueden complementarse entre sí, aportando información adicional o confirmatoria; adecuándose cada una a la situación clínica específica y apoyándose en su propia fortaleza evitando o supliendo debilidades de las otras. Para abordar el tema de la MI en la IC es conveniente revisar los diferentes aspectos referentes al diagnóstico de función sistodiastólica; al diagnóstico etiológico, pronóstico y a la evaluación terapéutica.

\section{Función sistólica y diastólica}

El ETT es el primer estudio que nos aportará datos de la función sistólica y diastólica. La FEVI y la estimación de las presiones de llenado cardíaco son los elementos más relevantes en la toma de decisiones terapéuticas.

Se acepta actualmente una clasificación de la IC según el rango de FEVI ${ }^{(1)}$ :

- IC con FEVI preservada (ICFEp): presencia de síntomas y signos; FEVI $\geq 50 \%$; BNP elevado y daño estructural relevante (HVI, AI dilatada) o disfunción diastólica.

- IC con FEVI intermedia (ICFEi): presencia de síntomas y signos; FEVI 40\%-49\%; BNP elevado y daño estructural relevante (HVI, AI dilatada) o disfunción diastólica.

- IC con FEVI reducida (ICFEr): presencia de síntomas y signos; FEVI $<40 \%$. 
Las nuevas técnicas ecocardiográficas (Doppler tisular y SLG) permiten, como ya fue dicho, detectar disfunción sistólica previa a la disminución de la FEVI, y se deben aplicar siempre que se plantee ICFEp $^{(1,2)}$. En caso de mala ventana, la CR es la alternativa para definir tanto la FEVI como la función sistólica del VD.

El abordaje de la disfunción diastólica es aún tema de debate. El concepto es complejo y está pobremente sistematizado. En todos los casos un hecho esencial es el aumento de la PDFVI y los esfuerzos se centran en su adecuada evaluación ${ }^{(1,2,21)}$.

Se reconocen tres situaciones que implican diferentes criterios ecocardiográficos relacionados con las presiones de llenado del VI:

- Evaluación de la PDFVI en pacientes con FEVI preservada.

- Evaluación de la PDFVI en pacientes con FEVI disminuida.

- Evaluación de la PDFVI en situaciones especiales.

\subsection{Evaluación de la PDFVI en pacientes con ICFEp}

Debe basarse, esencialmente, en los criterios ecocardiográficos explicados en el apartado 1. La Sociedad Americana de Ecocardiografía y la Asociación Europea de Imagenología Cardiovascular proponen un algoritmo utilizando en forma sistematizada dichos criterios para aumentar la especificidad diagnóstica del método, si bien este algoritmo no ha sido validado aún (figura 1)(21).

Si los parámetros cumplen con el criterio de indeterminado según el algoritmo, se debe dar mayor valor a la relación E/e', de manera tal que si la misma es $<8$ se admite que las presiones de llenado son normales y no se plantea disfunción diastólica, pero si es $>8$ pero $<14$ será necesario integrar la valoración estructural (sobre todo el volumen de la aurícula izquierda) para apoyar la presencia de disfunción diastólica.

\subsection{Evaluación de la PDFVI en pacientes con ICFEr}

En el caso de ICFEr la evaluación de la PDFVI se basa, fundamentalmente, en el patrón de llenado del VI obtenido por Doppler pulsado a nivel de punta de válvula mitral (relación velocidad de onda E y A), y tan solo en forma accesoria en los otros cuatro signos determinantes de las presiones del VI mencionados.

\subsection{Evaluación de la PDFVI en situaciones especiales}

Existen situaciones en las cuales los signos ecocardiográficos de PDFVI están alterados y se requiere de algoritmos propios, como en los casos de fibrilación auricular (FA) o las valvulopatías mitrales.
La FA es muy frecuente tanto en la ICFEp como en la ICFEr. En la presencia de FA los parámetros para medir PDFVI son diferentes a los antedichos. Los de mayor sensibilidad, en estos casos, son la aceleración máxima de velocidad E mitral y el tiempo de relajación isovolumétrica (IVRT). Otros parámetros útiles son una velocidad diastólica venosa pulmonar y un índice $\mathrm{E} / \mathrm{e}^{\prime(21,35)}$.

En pacientes con insuficiencia mitral los parámetros recomendados para la valoración de PDFVI son: el IVRT; el índice E/e'; la diferencia entre la velocidad A reversa en venas pulmonares y la velocidad de onda A del flujo mitral, con el punto de corte en $30 \mathrm{mseg}^{(21,36)}$.

Si bien la ecocardiografía es el método más utilizado para valorar presiones de llenado y disfunción diastólica, en casos especiales por su gravedad o en definiciones especiales, como los candidatos a trasplante cardíaco, lo que corresponde es la medida directa de las presiones de llenado a través de un estudio hemodinámico invasivo.

\section{Diagnóstico etiológico y evaluación de pronóstico}

La ETT sigue siendo el paso inicial para definir la etiología de la IC mediante una rápida valoración de la morfología cardíaca y estimación de la FEVI. La valoración morfológica permite detectar posibles cardiopatías congénitas o patologías valvulares primarias que justifiquen los síntomas del paciente.

La CR, sin embargo, ha cobrado un papel interesante en la valoración etiológica a través de sus secuencias de caracterización tisular y, sobre todo, el estudio del realce tardío. Permite separar la cardiopatía isquémica de otras cardiopatías no isquémicas similares en su presentación ecocardiográfica y clínica; permite orientar a algunas etiologías particulares de miocardiopatía y, finalmente, la presencia de realce tardío tiene, en sí mismo, valor de mal pronóstico.

Hay situaciones clínicas particulares que requieren un abordaje de MI para el diagnóstico, valoración etiológica y pronóstico diferencial; se destacarán los principales escenarios.

\subsection{Cardiomiopatía isquémica}

La etiología isquémica es la más prevalente (60\%-70\%) y la de peor pronóstico en los pacientes con ICFEr, pero puede ser también potencialmente reversible ${ }^{(1,39)}$. En la evaluación de la etiología isquémica de la IC es, quizá, donde se torna más útil el concepto de MI. Los dos fenómenos esenciales a valorar con implicancias terapéuticas son: la presencia de isquemia y la presencia de viabilidad miocárdica. 


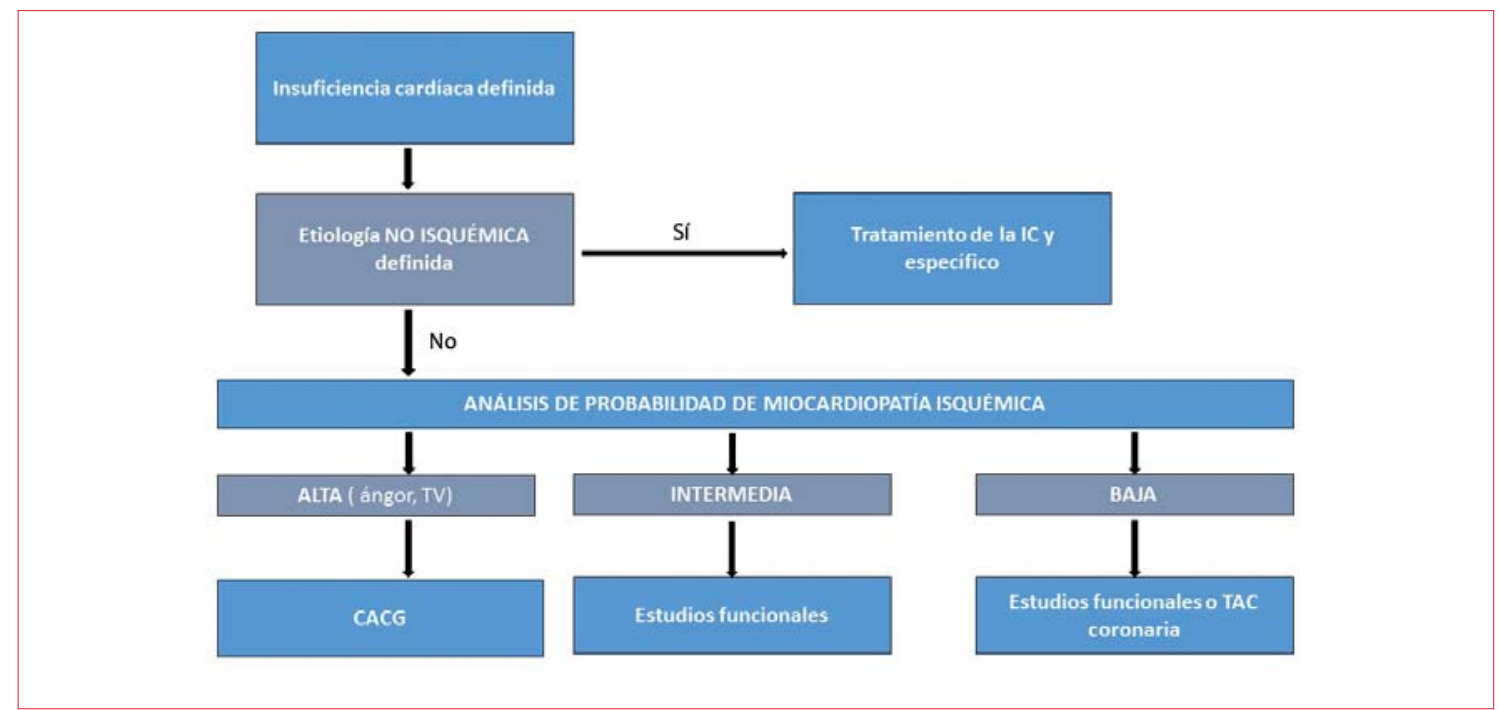

Figura 2. Algoritmo diagnóstico para valoración de etiología isquémica en la insuficiencia cardíaca. IC: insuficiencia cardíaca; CACG: cineangiocoronariografía; TV: taquicardia ventricular; TAC: tomografía axial computarizada.

La modalidad de imagen a seguir va a depender de la probabilidad pretest de cada paciente de presentar ateromatosis coronaria. Si la probabilidad es baja, no es posible plantear otra etiología y persiste la sospecha de coronariopatía, se puede optar por descartar la misma con un método de evaluación anatómica con alto valor predictivo negativo (VPN), como la TC coronaria, o realizar un test funcional de isquemia. Si este último es positivo y de moderado a alto riesgo se debe realizar cineangiocoronariografía (CACG), pero si es de bajo riesgo o presenta resultados discordantes o dudosos, y persiste la sospecha de coronariopatía, es razonable pasar a la valoración coronaria no invasiva (TC coronaria) como modo de descartar esta entidad.

En los pacientes con probabilidad intermedia es recomendable realizar un test funcional de isquemia y seguir con igual criterio que la situación anterior.

En los pacientes con alta probabilidad de coronariopatía y alto riesgo (ángor, arritmias ventriculares) se justifica la CACG como primera opción $^{(1,23,40)}$. En la figura 2 se resumen estos conceptos.

Respecto al análisis de la viabilidad miocárdica todos los métodos aportan distintos aspectos en cuanto a la presencia de tejido viable (hibernado) y no viable (necrosis-fibrosis), pues analizan enfoques fisiopatológicos diferentes y, además, todos varían en su sensibilidad y especificidad al ser evaluados en base a la recuperación funcional posrevascularización.

Aquellas pruebas basadas en la perfusión (tomografía por emisión de positrones-spect) han demostrado mayor sensibilidad que especificidad, y las pruebas que evalúan la respuesta contráctil frente a un inotrópico o esfuerzo (ecocardiografía de estrés) presentan mayor especificidad que sensibili$\operatorname{dad}^{(1,42,43)}$.

El método que presenta mayor sensibilidad y especificidad al momento de evaluar isquemia y viabilidad es la CR con estrés. Reúne una excelente resolución de imagen sumado a la posibilidad de realizar análisis con dobutamina o adenosina, o ambas (valorando alteraciones sectoriales y de la perfusión), y la posibilidad final de valorar el RT para definir viabilidad (alta sensibilidad y especificidad para recuperación funcional si el realce es menor al $25 \%$ del espesor parietal total y muy baja si ocupa más del $75 \%)^{(1,44)}$.

Si bien la CR es el estudio más completo para evaluar viabilidad, sin irradiación del paciente, su logística compleja en costos y accesibilidad no lo hacen el método de elección, quedando postergado a la disponibilidad del medio.

Otro método a considerar es la tomografía por emisión de positrones (PET) por su excelente resolución espacial y temporal y la capacidad de separar y cuantificar el miocardio necrótico y el hibernado usando trazador de perfusión y metabólico. Este método, por los mismos motivos que la CR, sumado al hecho de que es un método irradiante, no se ha extendido en la práctica cardiológica cotidiana ${ }^{(42)}$.

\subsection{Miocardiopatía dilatada}

La miocardiopatía dilatada (MCD) se define por la presencia de dilatación y disfunción del VI o VD, o ambos, que no pueda ser explicada por la presencia de coronariopatía, alteraciones congénitas, valvulopatías o hipertensión arterial ${ }^{(1)}$. Por tanto, en su va- 

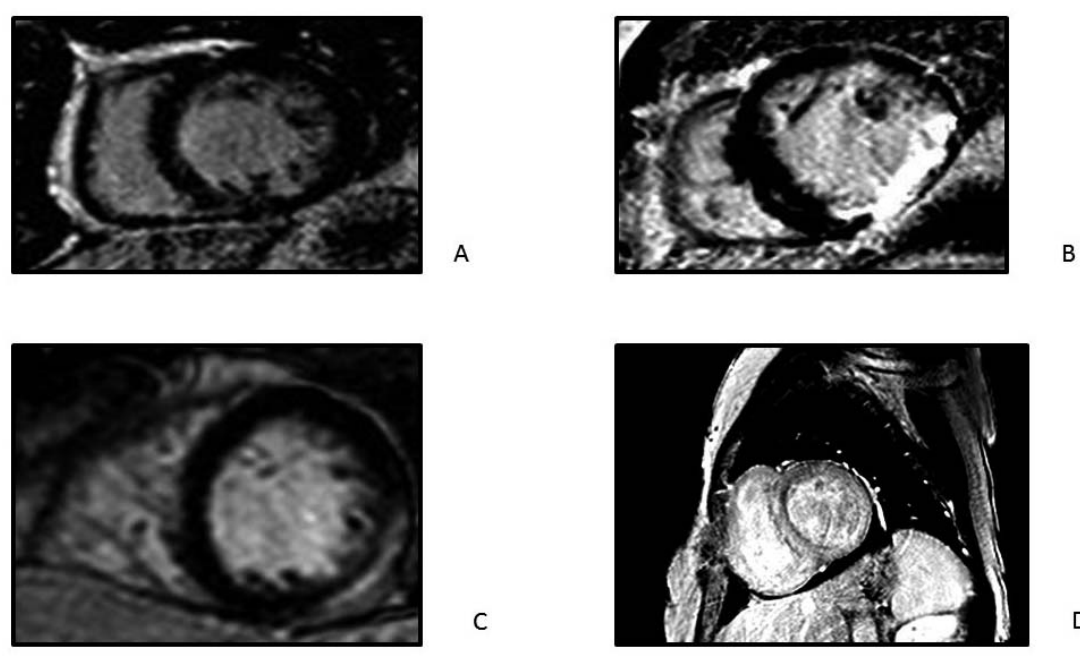

C

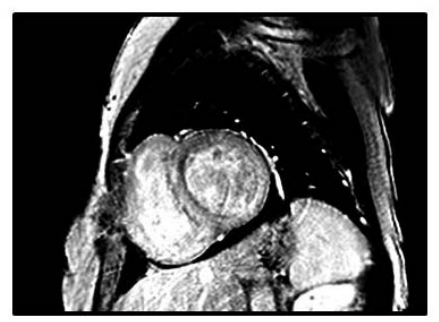

D

Figura 3. Patrones de realce tardío en búsqueda etiológica. A: normal. B: tipo isquémico (subendocárdico o transmural); C: tipo no isquémico (paciente con miocarditis); D: patrón de realce tardío endocárdico global (paciente con amiloidosis cardíaca).

loración se deben excluir todas estas posibilidades etiológicas a través de la clínica, el ECG, la radiografía de tórax y la ETT. En la búsqueda de la causa isquémica valen los conceptos vertidos en el epígrafe anterior.

La caracterización tisular de la CR y la evaluación del patrón de fibrosis y su ubicación, mediante la secuencia de realce tardío, pueden ser útiles ante la sospecha de enfermedades infiltrativas o inflamatorias (figura 3).

La CR es útil y superior a la ETT en la valoración estructural del VI no compactado o la miocardiopatía arritmogénica con criterios diagnósticos que han aumentado la especificidad del méto$\mathrm{do}^{(1,44-46)}$

La MI tiene utilidad en la valoración pronóstica de la MCD, ya sea por los datos de la ETT aceptados como predictores independientes de mal pronóstico (FEVI, volumen telesistólico, volumen de la AI, etcétera $^{(47)}$, o por los aportados por la CR como la presencia de realce tardío o la evaluación del T1 mapping; estos dos últimos han demostrado ser predictores independientes muy fuertes de muerte súbita y eventos adversos ${ }^{(1,47-50)}$.

\subsection{Miocardiopatías restrictivas}

La MI es muy importante en el diagnóstico de las miocardiopatías restrictivas (MR). Se pueden describir dos "fenotipos" imagenológicos de las MR: aquellas con aumento del espesor miocárdico y disfunción ventricular, dentro de las cuales la más frecuente es la amiloidosis, y un grupo de patologías menos frecuentes, que en forma primaria generan dilatación y disfunción ventricular (sarcoidosis, hemocromatosis, enfermedad de Wegener) ${ }^{(51)}$.
En todo paciente en el que se plantee el diagnóstico de amiloidosis o presente una miocardiopatía con aumento del espesor parietal debe ser considerada la complementariedad de realizarse un ecocardiograma y $\mathrm{CR}^{(1,51-53)}$.

La CR es la técnica de mayor utilidad en la valoración de la amiloidosis cardíaca al permitir afirmar el diagnóstico, descartar diferenciales, estimar pronóstico y diferenciar el tipo de amiloidosis ${ }^{(53,54)}$.

\section{Diagnóstico de evaluación terapéutica}

El monitoreo de la función sistodiastólica por imagen es fundamental para la evaluación terapéutica de cualquier paciente con IC.

El conocimiento de la FEVI define el uso de fármacos que han demostrado mejorar la sobrevida de pacientes con ICFEr, como los IECA/ARAII, los betabloqueantes, antagonistas de la aldosterona, etcétera.

La valoración de la función diastólica, que varía con el grado de descompensación de la IC, complementa la indicación de diuréticos y ayuda a definir altas hospitalarias.

La MI colabora en resoluciones terapéuticas, ya sea con la búsqueda de viabilidad para definir revascularización miocárdica en la cardiomiopatía isquémica o al aportar hechos imagenológicos para una mejor realización de procedimientos como la resincronización cardíaca.

En cuanto a la terapia de resincronización cardíaca (TRC), para la cual es un prerrequisito la presencia de disfunción sistólica con FEVI $\leq 35 \%$, aún permanece un porcentaje significativo de pacientes que no muestran la respuesta esperada. Si bien la ecografía y la CR a través del tagging pueden valo- 


\section{Resumen conceptual}

- Los estudios de imagen son claves en la evaluación de pacientes con IC, tanto en sus etapas diagnósticas iniciales como en la estimación del pronóstico y control del tratamiento farmacológico o de tratamientos avanzados no farmacológicos.

- La ecocardiografía transtorácica bidimensional es el método más importante para valorar pacientes con sospecha de IC por la cantidad de información morfológica, funcional y hemodinámica brindada en forma no invasiva, sin iatrogenia y a bajo costo.

- Se deben considerar nuevas técnicas ecocardiográficas (Doppler tisular y strain) en situaciones clínicas de alto riesgo de IC para identificar disfunción miocárdica sistólica precoz.

- Otras técnicas de imagen complementarias sirven como alternativa en caso de mala ventana ecocardiográfica y responden a preguntas específicas en algunas cardiopatías.
- La resonancia cardíaca a través de la secuencia de realce tardío diferencia la etiología isquémica de la no isquémica y dentro de esta última orienta a la etiología según el patrón de distribución; además, en caso de ser positivo, marca mal pronóstico. Permite seleccionar mejor a los pacientes y asesorar en la terapia de resincronización cardíaca.

- La tomografía cardíaca tiene su fortaleza en descartar enfermedad coronaria en pacientes con baja-moderada probabilidad de tenerla.

- Concebir los estudios de imagen desde el concepto de multimodalidad, coordinando las técnicas según sus fortalezas y debilidades y en función de las necesidades de cada caso clínico, constituye un gran aporte para el manejo de los pacientes con IC. rar la disincronía, todavía no han logrado identificar entre los candidatos a aquellos que se comportan respondiendo positivamente (respondedores) a la TRC.

Se puede afirmar que los pacientes con cardiomiopatía isquémica con extensas áreas de necrosis son malos candidatos a TRC. A su vez, el lugar de esa zona necrosada es importante para definir la respuesta, pues si la misma se encuentra en el lugar de aposición del electrodo de resincronización, no va a haber buena respuesta contráctil.

La CR, a través de la secuencia de realce tardío, permite definir aquellos pacientes que son malos candidatos a TRC por extensas áreas necrosadas, pero puede, también, ayudar a la planificación de la colocación del dispositivo topografiando las áreas de necrosis $^{(55,56)}$.

Un paso limitante del procedimiento de resincronización es la colocación del electrodo del VI en una rama del seno coronario. Para ello el conocimiento de la anatomía venosa coronaria es vital; esta se puede conocer mediante flebografía invasiva pero también a través de flebografía no invasiva por tomografía cardíaca multicorte ${ }^{(73)}$, o por CR (con el beneficio de la ausencia de radiación, aunque no ha sido validada aún en la práctica clínica).

\section{III) Perspectivas futuras}

Los estudios de imagen cardíaca son claves en la integración del diagnóstico, pronóstico y tratamiento de la IC. Seguramente se seguirá consolidando el proceso de validación de las técnicas que han sido mencionadas en este capítulo. Se vislumbra, en un futuro cercano, el uso en la práctica diaria de técnicas de fusión, tanto anatómicas como funcionales.

La ecocardiografía es y será por ahora la técnica de imagen central para la valoración de la IC por su alta disponibilidad, ausencia de iatrogenia potencial, economía y alta reproducibilidad; sumado a las nuevas técnicas (deformación y ecocardiografía tridimensional) que prometen alto impacto clínico de la mano de su mayor difusión, mejora y unificación de softwares.

Es relevante el desarrollo de técnicas de imagen que se fusionan para el diagnóstico y mejor tratamiento de algunas causas de IC, como la fusión de la ETT bi y tridimensional con la radioscopía en cardiología intervencionista para el tratamiento de cardiopatías estructurales ${ }^{(1,40)}$.

Para la TC es esperable su consolidación en las cardiopatías estructurales y grandes vasos como guía a la terapéutica intervencionista, tanto en la evaluación previa de la propia cardiopatía y también como referencia anatómica en implantes y complicaciones de dispositivos para la IC avanzada (TRC, cardiodesfibrilador implantable, asistencia ventricular mecánica). Se está profundizando, en el área de la investigación, la asociación de la imagen anatómica con la valoración fisiológica para evaluar isquemia, evaluándose en TC de perfusión con estrés y TC con FFR (reserva de flujo funcional)(1,40,41,58).

La CR ha ganado un rol protagónico en la valoración de la IC fundamentalmente por las secuencias que permiten caracterizar el tejido ${ }^{(1,40)}$. Son te- 
mas de investigación, técnicas complementarias como la tractografía con tensor de difusión, que analiza la compleja ultraestructura del miocardio a través de la orientación de las fibras cardíacas mediante el análisis de la autodifusión de las moléculas de agua de cada fibra, permitiendo un mapeo del movimiento de estas moléculas ${ }^{(40,58,59)}$.

\section{Bibliografía}

1. Ponikowski P, Voors AA, Anker SD, Bueno H, Cleland JG, Coats AJ, et al. 2016 ESC Guidelines for the diagnosis and treatment of acute and chronic heart failure: The Task Force for the diagnosis and treatment of acute and chronic heart failure of the European Society of Cardiology (ESC). Developed with the special contribution of the Heart Failure Association (HFA) of the ESC. Eur J Heart Fail 2016; 18(8):891-975.

2. Lang RM, Badano LP, Mor-Avi V, Afilalo J, Armstrong A, Ernande L, et al. Recommendations for cardiac chamber quantification by echocardiography in adults: an update from the American Society of Echocardiography and the European Association of Cardiovascular Imaging. Eur Heart J Cardiovasc Imaging 2015; 16(3):233-70.

3. Cole GD, Dhutia NM, Shun-Shin MJ, Willson K, Harrison J, Raphael CE, et al. Defining the real-world reproducibility of visual grading of left ventricular function and visual estimation of left ventricular ejection fraction: impact of image quality, experience and accreditation. Int $\mathrm{J}$ Cardiovasc Imaging 2015; 31(7):1303-14.

4. Knackstedt C, Bekkers SC, Schummers G, Schreckenberg M, Muraru D, Badano LP, et al. Fully automated versus standard tracking of left ventricular ejection fraction and longitudinal strain: the FAST-EFs multicenter study. J Am Coll Cardiol 2015; 66(13):1456-66

5. Kühl HP, Schreckenberg M, Rulands D, Katoh M, Schäfer W, Schummers G, et al. High-resolution transthoracic real-time three-dimensional echocardiography: quantitation of cardiac volumes and function using semi-automatic border detection and comparison with cardiac magnetic resonance imaging. J Am Coll Cardiol 2004; 43(11):2083-90.

6. Corsi C, Lang RM, Veronesi F, Weinert L, Caiani EG, MacEneaney P, et al. Volumetric quantification of global and regional left ventricular function from real-time three-dimensional echocardiographic images. Circulation 2005; 112(8):1161-70.

7. Shimada YJ, Shiota T. A meta-analysis and investigation for the source of bias of left ventricular volumes and function by three-dimensional echocardiography in comparison with magnetic resonance imaging. Am J Cardiol 2011; 107(1):126-38.
8. Chang SA, Lee SC, Kim EY, Hahm SH, Jang SY, Park SJ, et al. Feasibility of single-beat full-volume capture real-time three-dimensional echocardiography and auto-contouring algorithm for quantification of left ventricular volume: validation with cardiac magnetic resonance imaging. J Am Soc Echocardiogr 2011; 24(8):853-9.

9. Nikitin NP, Constantin C, Loh PH, Ghosh J, Lukaschuk EI, Bennett A, et al. New generation 3-dimensional echocardiography for left ventricular volumetric and functional measurements: comparison with cardiac magnetic resonance. Eur J Echocardiogr 2006; 7(5):365-72.

10. Vinereanu D, Lim PO, Frenneaux MP, Fraser AG. Reduced myocardial velocities of left ventricular long-axis contraction identify both systolic and diastolic heart failure-a comparison with brain natriuretic peptide. Eur J Heart Fail 2005; 7(4):512-9.

11. Poulsen SH, Andersen NH, Ivarsen PI, Mogensen CE, Egeblad H. Doppler tissue imaging reveals systolic dysfunction in patients with hypertension and apparent "isolated" diastolic dysfunction. J Am Soc Echocardiogr 2003; 16(7):724-31.

12. Dandel M, Hetzer R. Echocardiographic strain and strain rate imaging-clinical applications. Int J Cardiol 2009; 132(1):11-24.

13. Cianciulli T, Prieto O, Desseno A, Pedri S. Deformación miocárdica. En: Cianculli T, Prezioso H, Lax L. Nuevas técnicas ecocardiográficas. Buenos Aires: Ediciones Journal, 2012:15-49.

14. Mondillo S, Galderisi M, Mele D, Cameli M, Lomoriello VS, Zacà V, et al; Echocardiography Study Group of The Italian Society of Cardiology (Rome, Italy). Speckle-tracking echocardiography: a new technique for assessing myocardial function. J Ultrasound Med 2011; 30(1):71-83.

15. Kraigher-Krainer E, Shah AM, Gupta DK, Santos A, Claggett B, Pieske B, et al. Impaired systolic function by strain imaging in heart failure with preserved ejection fraction. J Am Coll Cardiol 2014; 63(5):447-56.

16. Thomas JD, Badano LP. EACVI-ASE-industry initiative to standardize deformation imaging: a brief update from the co-chairs. Eur Heart J Cardiovasc Imaging 2013; 14(11):1039-40.

17. Parma G, Américo C, Dayan V, Lluberas N, Martínez F, Fajardo A, et al. Reportes de valores normales de deformación miocárdica en una población sana uruguaya. Rev Urug Cardiol 2016; 31(2):236-45.

18. Stanton T, Leano R, Marwick TH. Prediction of all-cause mortality from global longitudinal speckle strain: comparison with ejection fraction and wall motion scoring. Circ Cardiovasc Imaging 2009; 2(5):356-64. 
19. Mignot A, Donal E, Zaroui A, Reant P, Salem A, Hamon C, et al. Global longitudinal strain as a major predictor of cardiac events in patients with depressed left ventricular function: a multicenter study. J Am Soc Echocardiogr 2010; 23(10):1019-24.

20. Nagueh SF, Appleton CP, Gillebert TC, Marino PN, Oh JK, Smiseth OA, et al. Recommendations for the evaluation of left ventricular diastolic function by echocardiography. Eur J Echocardiogr 2009; 10(2):165-93.

21. Nagueh SF, Smiseth OA, Appleton CP, Byrd BF 3rd, Dokainish H, Edvardsen T, et al. Recommendations for the evaluation of left ventricular diastolic function by echocardiography: an update from the American Society of Echocardiography and the European Association of Cardiovascular Imaging. J Am Soc Echocardiogr 2016; 29(4):277-314.

22. Wang M, Yip G, Yu CM, Zhang Q, Zhang Y, Tse D, et al. Independent and incremental prognostic value of early mitral annulus velocity in patients with impaired left ventricular systolic function. J Am Coll Cardiol 2005; 45(2):272-7.

23. Nagueh SF, Middleton KJ, Kopelen HA, Zoghbi WA, Quiñones MA. Doppler tissue imaging: a noninvasive technique for evaluation of left ventricular relaxation and estimation of filling pressures. J Am Coll Cardiol 1997; 30(6):1527-33.

24. Kasner M, Westermann D, Steendijk P, Gaub $\mathbf{R}$, Wilkenshoff $\mathbf{U}$, Weitmann $\mathbf{K}$, et al. Utility of Doppler echocardiography and tissue Doppler imaging in the estimation of diastolic function in heart failure with normal ejection fraction: a comparative Doppler-conductance catheterization study. Circulation 2007; 116(6):637-47.

25. Otani K, Takeuchi M, Kaku K, Haruki N, Yoshitani H, Tamura M, et al. Impact of diastolic dysfunction grade on left atrial mechanics assessed by two-dimensional speckle tracking echocardiography. J Am Soc Echocardiogr 2010; 23(9):961-7.

26. Kurt M, Wang J, Torre-Amione G, Nagueh SF. Left atrial function in diastolic heart failure. Circ Cardiovasc Imaging 2009; 2(1):10-5.

27. Motoki H, Borowski AG, Shrestha K, Troughton RW, Martin MG, Tang WH, et al. Impact of left ventricular diastolic function on left atrial mechanics in systolic heart failure. Am J Cardiol 2013; 112(6):821-6

28. Schulz-Menger J, Bluemke DA, Bremerich J, Flamm SD, Fogel MA, Friedrich MG, et al. Standardized image interpretation and post processing in cardiovascular magnetic resonance: Society for Cardiovascular Magnetic Resonance (SCMR) board of trustees task force on standardized post processing. $J$ Cardiovasc Magn Reson 2013; 15:35.

29. Kawel-Boehm N, Maceira A, Valsangiacomo-Buechel ER, Vogel-Claussen J, Turkbey
EB, Williams R, et al. Normal values for cardiovascular magnetic resonance in adults and children. $\mathrm{J}$ Cardiovasc Magn Reson 2015; 17:29.

30. Stuber M. Cardiovascular magnetic resonance tagging assessment of left ventricular diastolic function. En: Manning WJ, Pennell DJ. Cardiovascular magnetic resonance. 2 ed. Philadelphia: Saunder-Elsevier, 2010:69-75.

31. San Román JA, Soler Fernández R, Rodríguez García E, Fernández Avilés F. Conocimientos básicos necesarios para realizar resonancia magnética en cardiología. Rev Esp Cardiol Supl 2006; 6(E):7-14.

32. Sanz J, La Rocca G, Mireli JG. Mapeo miocárdico con resonancia magnética cardiaca: valor diagnóstico de las nuevas secuencias. Rev Esp Cardiol 2016; 69(9):849-61.

33. Andreini D, Pontone G, Pepi M, Ballerini G, Bartorelli AL, Magini A, et al. Diagnostic accuracy of multidetector computed tomography coronary angiography in patients with dilated cardiomyopathy. J Am Coll Cardiol 2007; 49(20):2044-50.

34. Greupner J, Zimmermann E, Grohmann A, Dübel HP, Althoff TF, Borges AC, et al. Head-to-head comparison of left ventricular function assessment with 64-row computed tomography, biplane left cineventriculography, and both 2- and 3-dimensional transthoracic echocardiography: comparison with magnetic resonance imaging as the reference standard. J Am Coll Cardiol 2012; 59(21):1897-907.

35. Nagueh SF, Kopelen HA, Quiñones MA. Assessment of left ventricular filling pressures by Doppler in the presence of atrial fibrillation. Circulation 1996; 94(9):2138-45.

36. Diwan A, McCulloch M, Lawrie GM, Reardon MJ, Nagueh SF. Doppler estimation of left ventricular filling pressures in patients with mitral valve disease. Circulation 2005; 111(24):3281-9.

37. Lichtenstein DA, Mezière GA. Relevance of lung ultrasound in the diagnosis of acute respiratory failure: the BLUE protocol. Chest 2008; 134(1):117-25.

38. Grenne B, Eek C, Sjøli B, Dahlslett T, Uchto M, Hol PK, et al. Acute coronary occlusion in non-ST-elevation acute coronary syndrome: outcome and early identification by strain echocardiography. Heart 2010; 96(19):1550-6.

39. Kindermann I, Barth C, Mahfoud F, Ukena C, Lenski M, Yilmaz A, et al. Update on myocarditis. J Am Coll Cardiol 2012; 59(9):779-92.

40. Gimelli A, Lancellotti P, Badano LP, Lombardi M, Gerber B, Plein S, et al. Non-invasive cardiac imaging evaluation of patients with chronic systolic heart failure: a report from the European Association of Cardiovascular Imaging (EACVI). Eur Heart J 2014; 35(48):3417-25. 
41. Mark DB, Berman DS, Budoff MJ, Carr JJ, Gerber TC, Hecht HS, et al. ACCF/ACR/AHA/ NASCI/SAIP/SCAI/SCCT 2010 expert consensus document on coronary computed tomographic angiography: a report of the American College of Cardiology Foundation Task Force on Expert Consensus Documents. J Am Coll Cardiol 2010; 55(23):2663-99.

42. Allman KC. Noninvasive assessment myocardial viability: current status and future directions. J Nucl Cardiol 2013; 20(4):618-37

43. Bax JJ, Poldermans D, Elhendy A, Boersma E, Rahimtoola SH. Sensitivity, specificity, and predictive accuracies of various noninvasive techniques for detecting hibernating myocardium. Curr Probl Cardiol 2001; 26(2):147-86.

44. Petersen SE, Selvanayagam JB, Wiesmann F, Robson MD, Francis JM, Anderson RH, et al. Left ventricular non-compaction: insights from cardiovascular magnetic resonance imaging. J Am Coll Cardiol 2005; 46(1):101-5.

45. Choi Y, Kim SM, Lee SC, Chang SA, Jang SY, Choe YH. Quantification of left ventricular trabeculae using cardiovascular magnetic resonance for the diagnosis of left ventricular non-compaction: evaluation of trabecular volume and refined semi-quantitative criteria. J Cardiovasc Magn Reson 2016; 18(1):24.

46. Marcus FI, McKenna WJ, Sherrill D, Basso C, Bauce B, Bluemke DA, et al. Diagnosis of arrhythmogenic right ventricular cardiomyopathy/dysplasia: proposed modification of the Task Force Criteria. Eur Heart J 2010; 31(7):806-14.

47. Carluccio E, Dini FL, Biagioli P, Lauciello R, Simioniuc A, Zuchi C, et al. The 'Echo Heart Failure Score': an echocardiographic risk prediction score of mortality in systolic heart failure. Eur J Heart Fail 2013; 15(8):868-76

48. Masci PG, Schuurman R, Andrea B, Ripoli A, Coceani M, Chiappino S, et al. Myocardial fibrosis as a key determinant of left ventricular remodeling in idiopathic dilated cardiomyopathy: a contrast-enhanced cardiovascular magnetic study. Circ Cardiovasc Imaging 2013; 6(5):790-9

49. Kuruvilla S, Adenaw N, Katwal AB, Lipinski MJ, Kramer CM, Salerno M. Late gadolinium enhancement on cardiac magnetic resonance predicts adverse cardiovascular outcomes in nonischemic car- diomyopathy: a systematic review and meta-analysis. Circ Cardiovasc Imaging 2014; 7(2):250-258

50. Puntmann VO, Carr-White G, Jabbour A, Yu CY, Gebker R, Kelle S, et al; International T1 Multicentre CMR Outcome Study. T1-Mapping and Outcome in Nonischemic Cardiomyopathy: all-cause mortality and Heart Failure. JACC Cardiovasc Imaging 2016; 9(1):40-50.

51. Seward JB, Casaclang-Verzosa G. Infiltrative cardiovascular diseases: cardiomyopathies that look alike. J Am Coll Cardiol 2010; 55(17):1769-79.

52. Sharma N, Howlett J. Current state of cardiac amyloidosis. Curr Opin Cardiol 2013; 28(2):242-8.

53. Fontana M, Chung R, Hawkins PN, Moon JC. Cardiovascular magnetic resonance for amyloidosis. Heart Fail Rev 2015; 20(2):133-44

54. Dungu JN, Valencia O, Pinney JH, Gibbs SD, Rowczenio D, Gilbertson JA, et al. CMR-based differentiation of AL and ATTR cardiac amyloidosis. JACC Cardiovasc Imaging 2014; 7(2):133-42.

55. De Winter O, Van de Veire $\mathbf{N}$, Van Heuverswijn F, Van Pottelberge G, Gillebert TC, De Sutter J. Relationship between QRS duration, left ventricular volumes and prevalence of nonviability in patients with coronary artery disease and severe left ventricular dysfunction. Eur J Heart Fail 2006; 8(3):275-7.

56. Ypenburg C, Schalij MJ, Bleeker GB, Steendijk P, Boersma E, Dibbets-Schneider P, et al. Extent of viability to predict response to cardiac resynchronization therapy in ischemic heart failure patients. J Nucl Med 2006; 47(10):1565-70.

57. Van de Veire NR, Schuijf JD, De Sutter J, Devos D, Bleeker GB, de Roos A, et al. Non-invasive visualization of the cardiac venous system in coronary artery disease patients using 64-slice computed tomography. J Am Coll Cardiol 2006; 48(9):1832-8.

58. Mishkin JD, Enriquez JR, Meyer DM, Bethea BT, Thibodeau JT, Patel PC, et al. Utilization of cardiac computed tomography angiography for the diagnosis of left ventricular assist device thrombosis. Circ Heart Fail 2012; 5(2):e27-9.

59. Axel L, Wedeen VJ, Ennis DB. Probing dynamic myocardial microstructure with cardiac magnetic resonance diffusion tensor imaging. J Cardiovasc Magn Reson 2014; 16:89. 